\title{
Level and distribution of secreted and cell-associated $N$-acetyl, $N$-glycolylneuraminic, and deaminoneuraminic acids in osteosarcoma cells isolated from patients
}

Thanyaluck Phitak ${ }^{\mathrm{a}}$, Jeerawan Klangjorhor ${ }^{\mathrm{a}}$, Peraphan Pothacharoen ${ }^{\mathrm{a}}$, Dumnoensun Pruksakorn ${ }^{\mathrm{b}}$, Prachya Kongtawelert ${ }^{\mathrm{a}, *}$

a Thailand Excellence Centre for Tissue Engineering and Stem Cells, Faculty of Medicine, Chiang Mai University, 110 Intravarorot Road, Sripoom, Chiang Mai 50200 Thailand

b Department of Orthopedics, Musculoskeletal Research Laboratory, Faculty of Medicine, Chiang Mai University, 110 Intravarorot Road, Sripoom, Chiang Mai 50200 Thailand

*Corresponding author, e-mail: prachya.kongtawelert@gmail.com

Received 3 Aug 2016

Accepted 27 Mar 2017

\begin{abstract}
Sialic acids, which are members of a family of 9-carbon carboxylated sugars, usually occupy the terminal position of the glycan chains of glycoconjugates and play an important role in various biological processes. $N$ acetylneuraminic acid (Neu5Ac) and $N$-glycolylneuraminic acid (Neu5Gc) are the dominant sialic acids in mammals, whereas deaminoneuraminic acids (KDN) can be detected in trace amounts. The increase of sialic acid levels has been reported in relation to the pathology and aggressiveness of cancer. Here we used a fluorometric-HPLC method to investigate the level and distribution of Neu5Ac, Neu5Gc, and KDN in osteosarcoma cells isolated from 7 patients and compare them with those in osteoblasts isolated from 3 patients with other bone diseases. Both cell types expressed all three sialic acids with abundances Neu5Ac $>$ Neu5Gc $>$ KDN. Total sialic acid levels in osteosarcoma cells were twice that in osteoblast cells. Only cell-associated Neu5Ac and Neu5Gc levels were higher, but not secreted forms. However, cell-associated and secreted KDN levels were not significantly different among those two groups. This suggests that the level and distribution of sialic acids in osteosarcoma cells were different from those in normal osteoblast cells. Thus their level, type, and distribution may be related to the pathology of osteosarcoma.
\end{abstract}

KEYWORDS: sialic acid, bone, cancer, HPLC

\section{INTRODUCTION}

Sialic acid (Sia) is a 9-carbon carboxylated sugar, named 2-keto-3-deoxy-D-glycero-D-galactononulosonic acid. Usually, Sia occupies the terminal position of the glycan chains of glycoconjugates (both glycoproteins and glycolipids), and plays an important role in various biological processes. It functions as a mediator for ligand-receptor and cell-cell interactions in fertilization, differentiation, and immunological and neurological events ${ }^{1}$. Occasionally, Sias are linked together to form a polymerized structure or polysialic acids. The most striking feature of Sia is its diversity. To date, more than 50 types of Sia have been characterized and they include derivatives of $N$-acetylneuraminic acid (Neu5Ac), $N$-glycolylneuraminic acid (Neu5Gc), and deaminoneuraminic acid (KDN, 2-keto-3-
deoxy-D-glycero-D-galacto-nononic acid), which are the three major backbones of $\mathrm{Sia}^{1}$. Other types include their derivatives that have been modified by acetylation, lactylation, methylation, and sulphation ${ }^{2}$. Basically, the most abundant Sia derivatives found in mammals are Neu5Ac and Neu5Gc. Owing to a human specific inactivating mutation in the cytidine monophosphate (CMP)Neu5Ac hydroxylase gene, which encodes the enzyme that catalyses CMP-Neu5Ac to CMPNeu5Gc, humans are incapable of synthesizing Neu5 $\mathrm{Gc}^{3}$; and therefore in normal human tissues and fluids, Neu5Gc is usually detected in very low amounts that are thought to originate from exogenous sources ${ }^{4,5}$.

The level of enzymes which transfer Sias to glycan chains (sialyltransferase) have been found to be reliable biomarkers of breast cancer activity, and 
serial measurements of this enzyme activity have been used to monitor the disease activity and the success or failure of the treatment ${ }^{6}$. Shamberger et al found that the level of Sia in the serum measured by colorimetric methods was increased in several types of cancers including bone cancer when compared with its level in normal serum. However, an increase in the Sia level was also found in the sera of patients with inflammatory diseases such as arthritis or Cronh's disease ${ }^{7}$. So far there have been thousands of scientific papers that have reported on the increase of the Sia level in many kinds of cancer cells and in secreted biological fluids from patients with various types of cancers.

Not only is the total level of Sia of relevance, but a specific derivative of increased Sia has also been identified in many types of cancer. The results showed different patterns of increased Sia derivatives depending on the type of cancer cell. The increased Sia types can be classified into 2 patterns; increase of human basic type of Sia (Neu5Ac), or increase of other types of Sias, such as Neu5Gc or $\mathrm{KDN}$, and especially for Neu5Gc which humans are unable to synthesize.

Yabu et al described the accumulation of free Neu5Ac-containing complex type $N$-glycan in pancreatic cancer ${ }^{8}$. They also investigated the accumulation of the same type of Sia in prostate cancers. Moreover, not only was the increase of free Neu5Ac-containing $\mathrm{N}$-glycans found, but also significant amounts of free $\mathrm{KDN}$-containing $\mathrm{N}$-glycans accumulated in prostate cancer tissues ${ }^{9}$. It was the first study that showed unequivocal chemical evidence for the occurrence of KDN glycoconjugates in human tissues. The alterations of the level and type of Sia might involve alteration of enzymes in Sia biosynthetic pathways or Sia transporters that uptake exogenous Sia into the cancer cells.

From the beginning of the Sia discovery by Levene, Landsteiner ${ }^{10}$ and Walz ${ }^{11}$ as a sugar-like component, until the discovery of its relationship to the pathology of cancer disease ${ }^{6}$, the molecular mechanism of this sugar-like molecule in cancer remains unknown. Many publications have suggested the role of Sia in cancer progression. In detail, the negative charge of Sia was believed to be involved in the metastatic process of cancer cells. Moreover, some reports have suggested that the elevated Sias are involved in the proliferation of cancer cells and the inhibition of enzymes involved in Sia biosynthesis resulting in a reduction in the proliferation and metastatic process of cancer cell lines ${ }^{12-14}$. Altogether, it could be suggested that enzymes involved in the Sia biosynthetic pathway could be used as a target for cancer therapy.

With regard to osteosarcoma, a malignant bone tumour consisting of cells with abnormal cellular functions, so far there have only been two reports that have investigated the relationship of the Sia level and type with the pathology of this cancer. The first one was published in Ref. 15 where it was reported that a high metastatic osteosarcoma cell line showed a significant elevation of total Sia when compared with that found in a low metastatic cell line. Moreover, not only was the basic type of Sia (Neu5Ac) found to increase but Neu5Gc also increased. The second publication studied the level of total Sia in the sera of 25 osteosarcoma patients. A significant elevation of the total Sia level was found when compared with that level in the control sera. However, an increase of the Sia level was also found in the sera of patients with other boneforming tumours ${ }^{16}$. Here, to better understand the relation of elevated Sia and the pathology of osteosarcoma, osteosarcoma cells were isolated from bone samples of 7 patients with osteosarcoma. The expression level and type of Sia were investigated in both the cell fraction and the cultured media using the fluorometric-HPLC method. Expression, distribution, and type of Sia in osteosarcoma cells were then compared with those in osteoblasts isolated from bones of patients with other bone diseases.

\section{MATERIALS AND METHODS}

\section{Isolation of osteoblast and osteosarcoma cells from bone samples of patients}

Bone samples were obtained with full informed consent from the arthroscopic diagnosis of osteosarcoma patients ( 7 cases) or other bone disease patients (3 cases) at Maharaj Nakorn Chiang Mai Hospital, Department of Orthopaedic, Faculty of Medicine, Chiang Mai University, Thailand (Ethical approval code: BIO-2556-01960).

Osteoblast cells were isolated from the cancellous bone samples by sequential collagenase-trypsin digestion. The soft connective tissue was removed from the outer surfaces of the bone samples. The bone fragments were minced into small pieces, washed with PBS ( $\mathrm{pH}$ 7.3) until no remaining haematopoietic marrow was visible and the bone fragments had assumed a white appearance. Osteoblasts were isolated with sequential collagenasetrypsin digestion by incubating the bone pieces in $5 \mathrm{mg} / \mathrm{ml}$ collagenase type I solution (Sigma Aldrich) and $0.25 \%$ trypsin solution (Sigma Aldrich) at $37^{\circ} \mathrm{C}$ 
with shaking for $20 \mathrm{~min}$ at each step. For osteosarcomas, cells were isolated from the bone samples by incubating the tissue in $5 \mathrm{mg} / \mathrm{ml}$ collagenase type I solution at $37^{\circ} \mathrm{C}$ for 1 day. After incubation, the supernatant was transferred into a new tube and centrifuged at $1200 \mathrm{rpm}$ for $10 \mathrm{~min}$. These pellets were gently re-suspended in DMEM supplemented with $10 \%$ foetal bovine serum (FBS, Sigma Aldrich), $100 \mathrm{IU} / \mathrm{ml}$ penicillin and $100 \mu \mathrm{g} / \mathrm{ml}$ streptomycin at $37^{\circ} \mathrm{C}$ in a humidified atmosphere of $5 \% \mathrm{CO}_{2}$. The medium was changed every 2 days, and the cells were subcultured when they reached confluence. The cells from the 2nd-4th passage were used for osteoblast characterization and other relevant experiments.

\section{Cell proliferation assay}

Osteoblasts and osteosarcoma cells were seeded at $10^{4}$ cells per well in 24-well-plates. At days 1, 2, $3,4,7,10,14,17,21,24$ and 28 , cell numbers were measured by trypsinization and counted using a haemacytometer. An early log-phase was used to estimate the population doubling time.

\section{Alkaline phosphatase (ALP) activity assay}

ALP activity was determined kinetically by monitoring the conversion of $p$-nitrophenyl phosphate ( $p$-NPP) to $p$-nitrophenol ${ }^{17}$. Osteoblasts and osteosarcoma cells were seeded at $10^{4}$ cells per well in 96-well-plates in four replications for each case. After $1,3,7,14,21$, and 28 days in the culture, the media of two wells were removed and the cells were washed with $100 \mu \mathrm{l}$ PBS. Eighty microlitres of $1 \mathrm{mg} / \mathrm{ml}$ phosphate substrate solution and $20 \mu \mathrm{l}$ of $1.5 \mathrm{M}$ alkaline buffer solution were added. The plate was incubated at $37^{\circ} \mathrm{C}$ for $30 \mathrm{~min}$ and the reaction was stopped by adding $50 \mu \mathrm{l}$ of $0.3 \mathrm{~N} \mathrm{NaOH}$ and absorbance was measured at $405 \mathrm{~nm}$. The cells in the other two wells were lysed with $20 \mu \mathrm{l}$ of $1 \%$ Triton X-100 in PBS to determine the protein content using Bradford assay kit (Bio-Rad). The activity was normalized to the protein content and was expressed as $\mu \mathrm{M} / \mu \mathrm{g}$ of protein $/ \mathrm{min}$.

\section{Alizarin red S histochemistry}

Osteoblasts and osteosarcoma cells were seeded at $5 \times 10^{4}$ cells per well in 24-well-plates in four replications for each case. The cultured cells were stained with Alizarin red S on days 7, 14, 21, and 28 to assess the mineralized matrix ${ }^{18}$. The medium was removed, and the cell layers were rinsed with PBS 3 times and fixed in cold 95\% ethanol for $15 \mathrm{~min}$ at room temperature. Then the cell layers were washed with deionized water twice and allowed to air dry. The fixed cells were stained with $40 \mathrm{mM}$ Alizarin red S pH 4.1 (Sigma Aldrich). After $20 \mathrm{~min}$, the cell layers were washed with deionized water and observed both grossly and with a light microscope. An Alizarin red S stained monolayer was extracted to quantify the mineralized matrix based on the acetic acid extraction and neutralization with ammonium hydroxide and this was followed by colorimetric detection at $405 \mathrm{~nm}$.

\section{Osteogenic gene expression}

Total RNA of the cultured cells was extracted using an RNA extraction kit following the manufacturer's protocol (GE Healthcare, UK), and 500 ng of total RNA were converted to cDNA using RevertAid $\mathrm{H}$ minus first strand cDNA synthesis kit (Fermentus Life science, USA). For determination of osteogenic gene expression, Real-time RT-PCR was performed using commercial SsoFast EvaGreen Supermix dye according to the manufacturer's protocol (Bio-Rad, USA). The reactions were performed on the MiniOpticon Real-Time PCR Detection system (Bio-Rad, USA). Relative expression levels for each gene were normalized to the expression of GAPDH by the $2^{-\Delta \mathrm{CT}}$ method $^{19}$. The expressions of MMP-9, osteonectin (ON), collagen type I (Col I), collagen type X ( Col X), bone sialoprotein (BSP), and GAPDH mRNA levels were quantified to show the osteoblastic characteristics ${ }^{20}$. The sequences of used primers were shown in Table 1.

\section{Cells and cultured media preparation for Sia analysis}

Osteoblasts or osteosarcoma cells were cultured in 6-well plates until $80 \%$ confluence was reached with changes of media every $48 \mathrm{~h}$. After reaching $80 \%$ confluence, the media was removed and the

Table 1 Sequences of the primers used in the experiments.

\begin{tabular}{|c|c|c|}
\hline Gene & Primer & Primer sequence \\
\hline MMP-9 & $\begin{array}{l}\text { forward } \\
\text { reverse }\end{array}$ & $\begin{array}{l}5^{\prime} \text {-TGAGAACCAATCTCACCGACAG - } 3^{\prime} \\
5^{\prime}-\text { TGCCACCCGAGTGTAACCAT }-3^{\prime}\end{array}$ \\
\hline ON & $\begin{array}{l}\text { forward } \\
\text { reverse }\end{array}$ & $\begin{array}{l}5^{\prime} \text {-TCCACAGTACCGGATTCTCTCT }-3^{\prime} \\
5^{\prime} \text { - TCTATGTTAGCACCTTGTCTCCAG - } 3^{\prime}\end{array}$ \\
\hline Col I & $\begin{array}{l}\text { forward } \\
\text { reverse }\end{array}$ & $\begin{array}{l}5^{\prime} \text { - CAGCCGCTTCACCTACAGC - } 3^{\prime} \\
5^{\prime} \text { - TTTTGTATTCAATCACTGTCTTGCC }-3^{\prime}\end{array}$ \\
\hline Col X & $\begin{array}{l}\text { forward } \\
\text { reverse }\end{array}$ & $\begin{array}{l}5^{\prime} \text {-AGCCAGGGTTGCCAGGACCA-3' } \\
5^{\prime} \text { - TTTTCCCACTCCAGGAGGGC - } 3^{\prime}\end{array}$ \\
\hline BSP & $\begin{array}{l}\text { forward } \\
\text { reverse }\end{array}$ & $\begin{array}{l}5^{\prime} \text {-GCAGTAGTGACTCATCCGAAGA }-3^{\prime} \\
5^{\prime} \text { - GCCTCAGAGTCTTCATCTTCATTC }-3^{\prime}\end{array}$ \\
\hline GAPDH & $\begin{array}{l}\text { forward } \\
\text { reverse }\end{array}$ & $\begin{array}{l}5^{\prime} \text {-GAAGGTGAAGGTCGGAGTC - }{ }^{\prime} \\
5^{\prime} \text {-GAAGATGGTGATGGGATTTC }-3^{\prime}\end{array}$ \\
\hline
\end{tabular}


culture cells were washed with PBS. Cells were continuously cultured in serum-free media for $24 \mathrm{~h}$.

Cultured media: four millilitres of cultured media (from 2 wells of a 6-well plate) were collected and concentrated using ultrafiltration disc YM-10 (Amicon Ultra). The concentration of proteins was measured using BCA Protein Assay Kit.

Cultured cells: $80 \%$ confluent cells were harvested from 2 wells of 6-well plates using a plastic cell scraper in PBS containing 2 mM EDTA after the cells were washed twice with cold PBS. After centrifugation at $850 \mathrm{~g}$ for $10 \mathrm{~min}$, the supernatant was removed and the cell pellets were disrupted by sonication for $10 \mathrm{~s} 3$ times on ice in $250 \mu \mathrm{l}$ lysis buffer containing proteinase inhibitors (10 mM Tris$\mathrm{HCl}, \mathrm{pH}$ 8.0). Part of the cell lysate was used for the quantitation of protein concentration using BCA Protein Assay Kit.

\section{Sample treatment and Sia analysis}

Both cell fractions and cultured media were hydrolysed with $0.1 \mathrm{~N}$ trifluoroacetic acid (TFA) at $80^{\circ} \mathrm{C}$ for $2 \mathrm{~h}$ to release free Sia from glycoconjugates. After hydrolysis, samples were purified using Strata C18E (Phenomenex, UK) to remove degraded proteins and other insoluble compounds. Purified samples were dried by speed vacuum. Sia in the samples or authentic KDN, Neu5Gc and Neu5Ac (kindly donated by Prof. Ken Kitajima, Laboratory of Animal Cell Function, Bioscience and Biotechnology Centre, Nagoya University) were derivatized with fluorescein 1,2diamino-4,5-methylenedioxybenzene (DMB). In brief, dried samples were reconstituted with $0.01 \mathrm{~N}$ TFA. Twenty microlitres of reconstituted sample was mixed with $20 \mu \mathrm{l}$ of $7 \mathrm{mM}$ DMB solution in 5.0 TFA containing $1 \mathrm{M}$ of 2-mercaptoethanol and $18 \mathrm{mM}$ sodium hydrosulphite, and incubated at $50^{\circ} \mathrm{C}$ for $2 \mathrm{~h}$. Five microlitres of the DMB derivative solution were diluted 5 times with Milli-Q water and directly applied to an ODS column (250 X $4.6 \mathrm{~mm}$ i.d., Luna 5u C18 100A, Phenomenex, UK) and eluted isocratically with acetonitrile/methanol/water $(9: 7: 84 \mathrm{v} / \mathrm{v} / \mathrm{v})$ with a flow rate of $1.0 \mathrm{ml} / \mathrm{min}$ at a column temperature of $26^{\circ} \mathrm{C}$ for $40-90 \mathrm{~min}$ on a Thermo Scientific HPLC system equipped with a Thermo FL-3000 fluorescence detector (excitation, $373 \mathrm{~nm}$; emission, $448 \mathrm{~nm}$ ). Retention times of Neu5Ac, Neu5Gc and KDN were compared with the authentic sample. The amount of each type of Sia was quantified using the area under its HPLC peak compared with the standard curve of the authentic sample.

\section{RESULTS}

\section{Characterization of osteosarcoma and osteoblast}

Osteosarcoma and osteoblast cells were isolated from the bone samples of 7 different patients diagnosed with osteosarcoma and 3 other patients diagnosed with other bone diseases at Maharaj Nakorn Chiang Mai Hospital, Thailand (Department of Orthopaedics, Faculty of Medicine, Chiang Mai University, Thailand). For the characterization of the isolated cells, previous studies have pointed out differences in the gene expression profiles of osteosarcoma cell lines and normal osteoblasts ${ }^{22-25}$. Moreover, using the immunological labelling technique, it was confirmed that there was a different expression of osteogenic markers including osteonectin (ON), bone sialoprotein (BSP) and collagen type I (Col I) which were expressed at lower levels in osteosarcoma as compared to osteoblasts ${ }^{20}$. MMP-9 and collagen type X ( $\mathrm{Col} \mathrm{X})$, metastatic and cartilage markers, respectively, were found to be highly expressed in osteosarcoma compared to their values of expression in osteoblasts ${ }^{20}$. Thus characterization of the isolated osteosarcoma and osteoblasts from the patients with respect to transcriptional expressions of MMP-9, Col X, Col I, ON and BSP was performed.

As shown in Fig. 1, the expressions of MMP-9 and Col $\mathrm{X}$ were higher in the osteosarcoma group than in the osteoblast group, whereas osteogenic markers (Col I, ON, BSP) were lower. Additionally, the population doubling time of osteosarcoma was significantly lower than that of osteoblast cells. These results suggest that, isolated, the osteoblasts and osteosarcoma cells show their characteristics in correct accordance with their diagnosis. Moreover, the ability to synthesize the mineralized matrix, a major capacity of bones, was also investigated by Alizarin red S staining as indicated in dark colour (Fig. 2). All isolated osteoblast samples showed significantly higher ability to synthesize mineralized matrix, especially at days 21 and 28 of the culture in the osteogenic media, compared to those of the isolated osteosarcoma samples. Along with the early markers of the mineralization process, alkaline phosphatase (ALP) activity was also measured (Fig. 3). It was found that in basal media, there was no difference of the ALP activity between the osteosarcoma and osteoblast groups. However, in the osteogenic media, which contained substances that induced the mineralization process, the ALP activity in the osteosarcoma group was significantly lower 

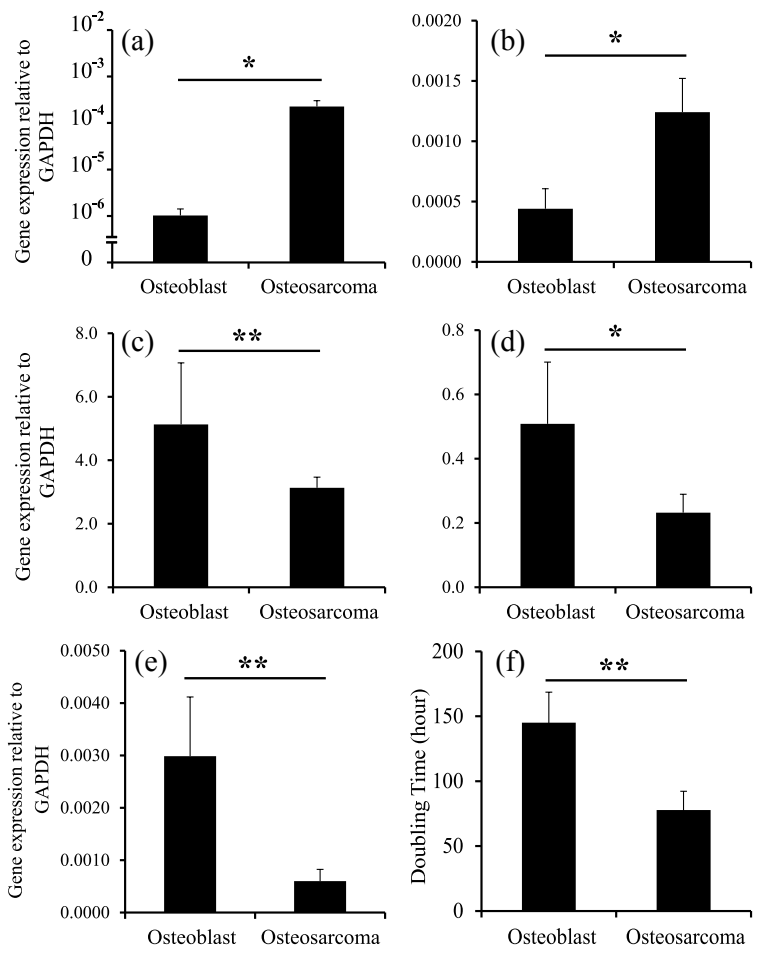

Fig. 1 The transcriptional level of (a) MMP-9, (b) Col X, (c) Col I, (d) ON, (e) BSP, and (f) doubling time of primary osteosarcoma and osteoblast cells isolated from 7 patients with osteosarcoma and 3 patients with other bone diseases, respectively. Isolated cells were cultured in basal media until reaching $80 \%$ confluence. Transcriptional expressions of those genes were measured by real time RT-PCT. *, ** Indicate significant difference calculated by independent $t$-test at $p<0.05$ and $p<0.01$, respectively.

than of that in the osteoblast group. It showed that the mineralization ability that is a major capacity of osteoblasts decreased in isolated osteosarcoma cells. This result indicated that the isolated cells of 10 patients were positively characterized as osteosarcoma and osteoblast according to their diagnosis.

\section{Detection of KDN, Neu5Gc and Neu5Ac using 1,2-diamino-4, 5-methylenedioxybenzene labelling-HPLC method}

There have been many reports suggesting methods to detect and quantify Sia (KDN, Neu5Gc, Neu5Ac). Since there are only trace amounts of Sias in human cells, thus in order to detect low quantities, Sias in the samples would be derivatized with fluorescein before separation and detection using the HPLC system. Here we set up a detection system according to the developed method by Yamakawa et $\mathrm{al}^{20,21}$. As shown in Fig. 4a, using this fluorometric-HPLC (a)
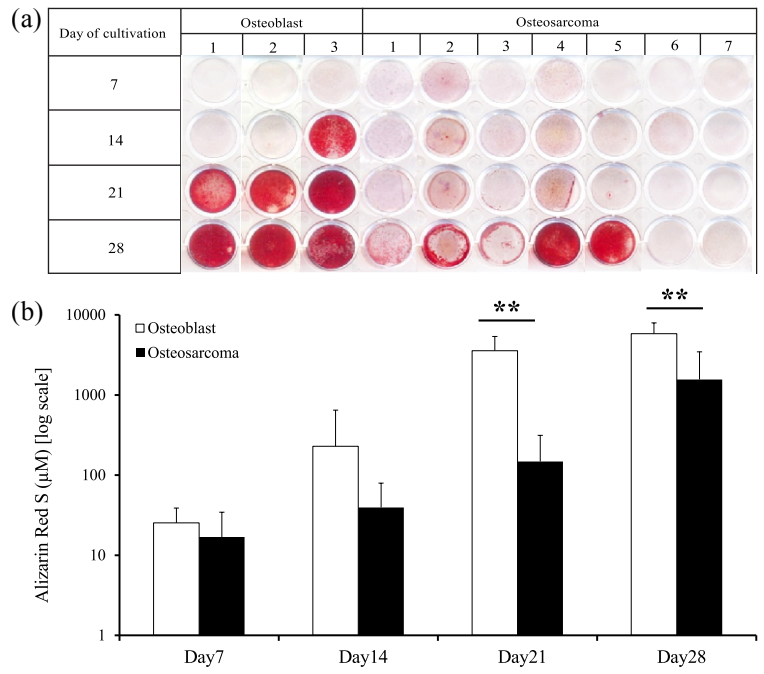

Fig. 2 Mineralized matrix of primary osteosarcoma and osteoblast cells isolated from patients with osteosarcoma and other bone diseases, respectively; (a) Alizarin red $S$ dye stained in the mineralized matrix (red), which indicates the samples at day 28 of the culture; (b) quantity of the stained Alizarin red $\mathrm{S}$ in the mineralized matrix in the osteosarcoma and osteoblasts cells at days 7, 14, 21, and 28 of the culture.
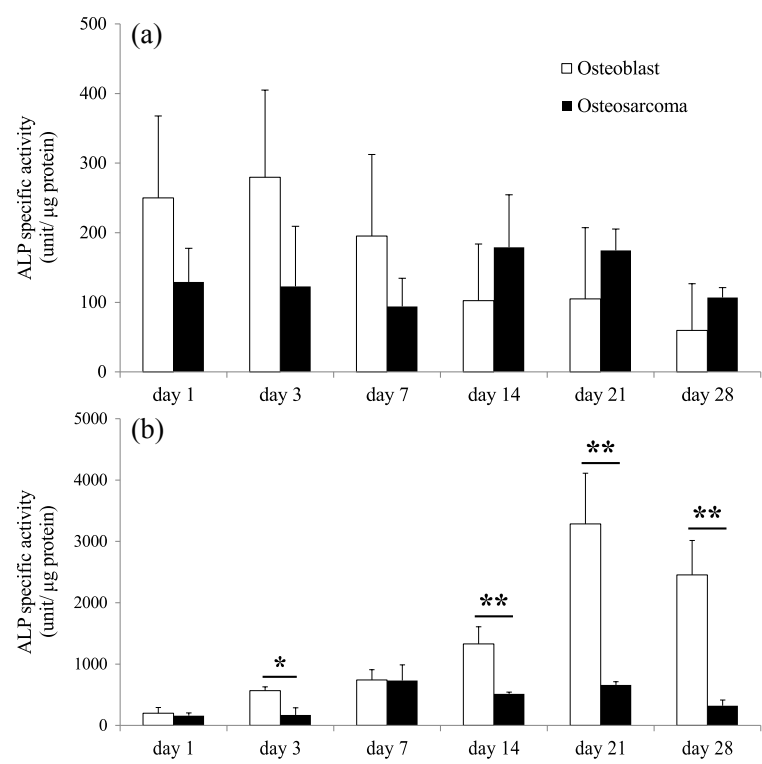

Fig. 3 The level of ALP activity in primary osteosarcoma and osteoblast cells isolated from patients with osteosarcoma and other bone diseases, respectively; ALP activity of the cells cultured (a) in the basal media, and (b) in osteogenic media at days $1,3,7,14,21$, and 28 of the culture. 


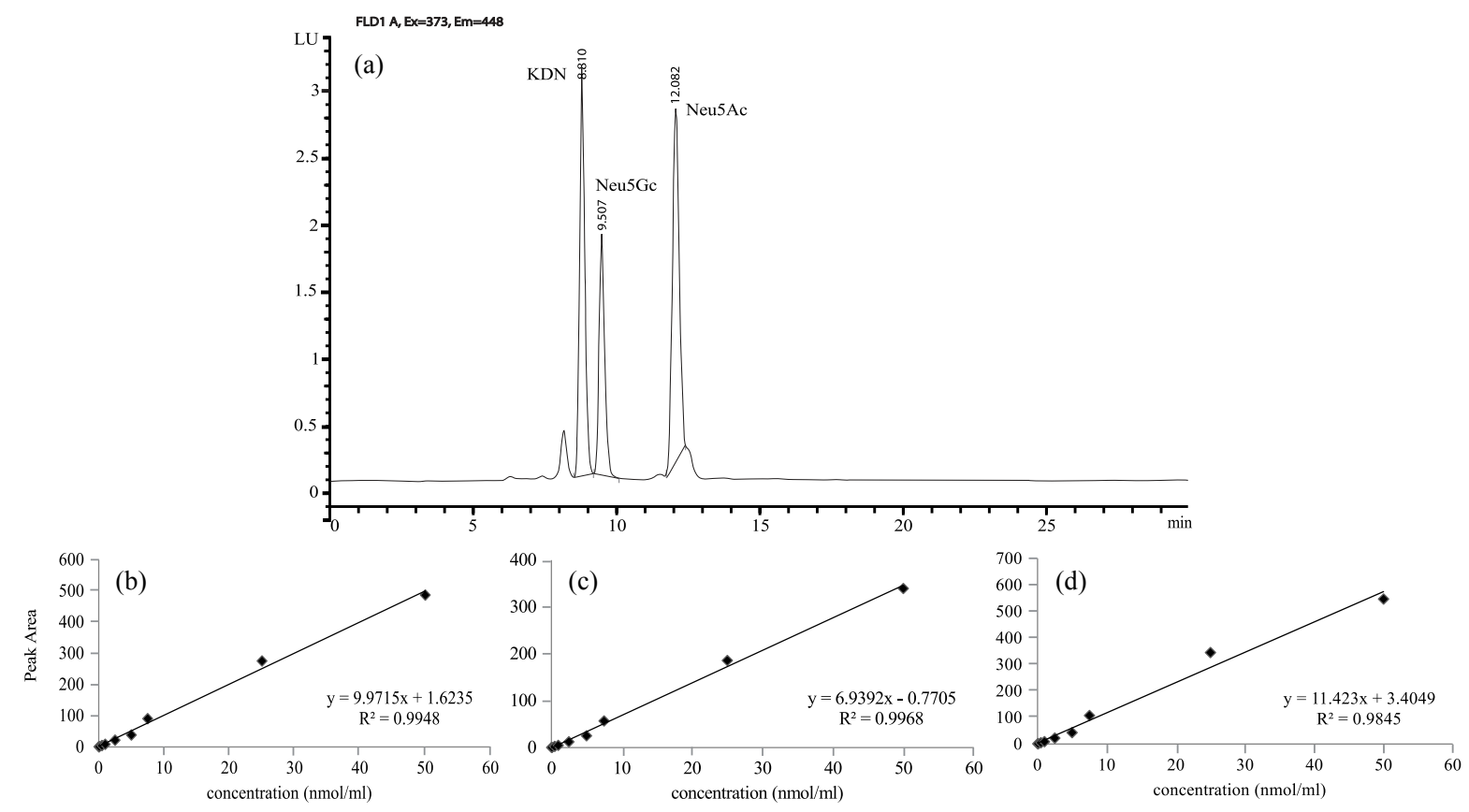

Fig. 4 Separation of authentic DMB-derivatized KDN, Neu5Gc, and Neu5Ac by HPLC; (a) chromatogram of authentic DMB-derivatized KDN, Neu5Gc, and Neu5Ac separated by HPLC; standard curve and correlation coefficient $\left(R^{2}\right)$ of the area under peak and concentration of (b) KDN, (c) Neu5Gc, and (d) Neu5Ac.

Table 2 Recovery rate of KDN, Neu5Gc, and Neu5Ac detected by fluorometric (DMB)-HPLC method.

\begin{tabular}{lc}
\hline Authentic sample & Recovery $(\%)^{\dagger}$ \\
\hline KDN & $100 \pm 14$ \\
Neu5Gc & $96 \pm 5$ \\
Neu5Ac & $95 \pm 8$ \\
\hline
\end{tabular}

$\dagger$ mean \pm SD

system KDN, Neu5Gc and Neu5Ac were separated with retention times of $8.8,9.5$, and $12.1 \mathrm{~min}$, respectively. Using authentic samples at concentrations of $0-50 \mathrm{nmol} / \mathrm{ml}$, the area under the peak correlated with their concentrations $\left(R^{2}>0.9\right)$, as shown in Fig. 4b-d, and the recovery percentages were more than $95 \%$ in all 3 authentic samples (Table 2). This result indicated that the set-up method was appropriate for the quantification of Sias in the samples.

\section{Level and distribution of secreted- and cell-associated KDN, Neu5Gc, and Neu5Ac}

Fig. 5 shows the average total amount of KDN, Neu5Gc and Neu5Ac (secreted plus cell-associated forms) in 7 samples of isolated osteosarcoma cells and in 3 samples of isolated osteoblasts. Neu5Ac was the major Sia found in the investigated cells.

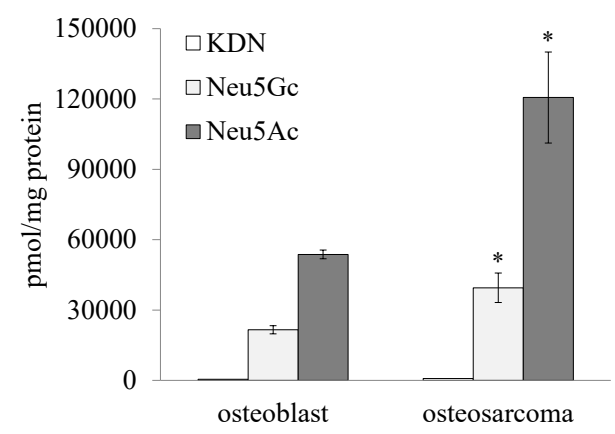

Fig. 5 Levels of total KDN, Neu5Gc and Neu5Ac in 7 samples of osteosarcomas and 3 samples of osteoblasts. * Indicates significant differences calculated by independent $t$-test at $p<0.05$. Values were expressed as mean $\pm \mathrm{SE}$ of 3 independent experiments.

Neu5Gc could also be detected and KDN was also detectable in trace amounts using this system. As shown in Fig. 5, and Table 3 (average amount in $\mathrm{pmol} / \mathrm{mg}$ protein), the average total amounts of all KDN, Neu5Gc and Neu5Ac in osteosarcoma were higher than those in the osteoblasts. However, only levels of Neu5Gc and Neu5Ac were significantly higher (Fig. 5). For the distribution of Sia, interestingly, only the amounts of cell-associated Neu5Gc and Neu5Ac, but not secreted Neu5Gc 
Table 3 Average levels of KDN, Neu5Gc and Neu5Ac in cell lysate and cultured media in 7 osteosarcoma samples and 3 osteoblast samples (pmol/mg protein and percentage of its total amount).

\begin{tabular}{lcccccccc}
\hline & \multicolumn{3}{c}{ Osteoblast } & & \multicolumn{3}{c}{ Osteosarcoma } \\
\cline { 2 - 3 } \cline { 7 - 9 } & KDN & Neu5Gc & Neu5Ac & & KDN & Neu5Gc & Neu5Ac \\
\hline Sialic acid in cell lysate (pmol/mg protein) & 175 & 4543 & 23058 & & 485 & 21145 & 81057 \\
(\% of total) & $(34)$ & $(21)$ & $(43)$ & & $(60)$ & $(54)$ & $(67)$ \\
Sialic acid in cultured media (pmol/mg protein) & 346 & 17063 & 30661 & & 327 & 18353 & 39564 \\
(\% of total) & $(66)$ & $(79)$ & $(57)$ & & $(40)$ & $(46)$ & $(33)$ \\
Total (pmol/mg protein) & 521 & 21606 & 53719 & & 812 & 39498 & 120621 \\
Cell lysate/media ratio & 0.5 & 0.3 & 0.8 & & 1.5 & & 1.2 & 2.1 \\
\hline
\end{tabular}
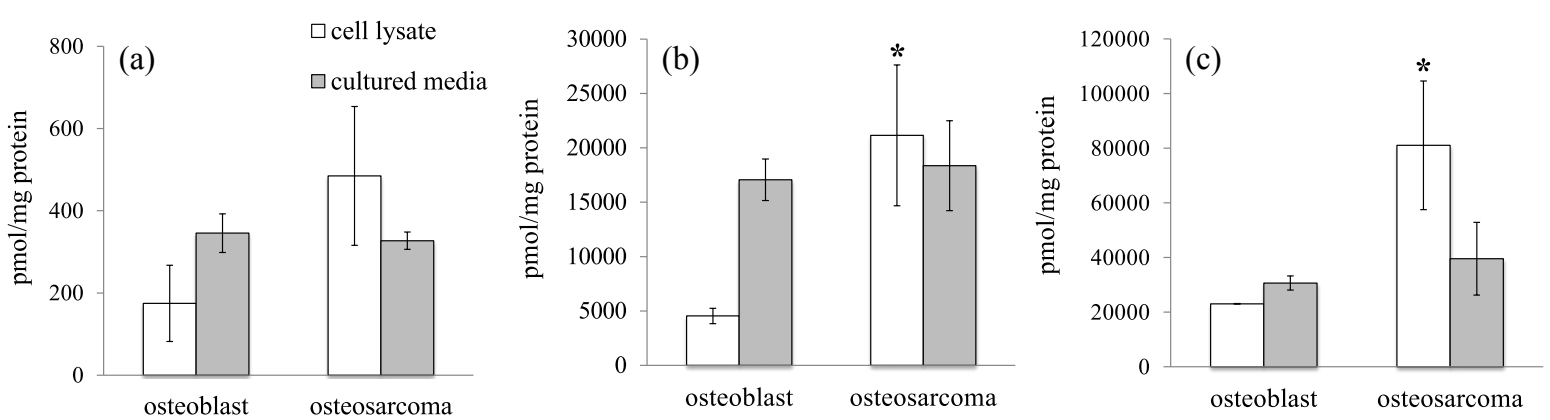

Fig. 6 Levels of (a) KDN, (b) Neu5Gc, and (c) Neu5Ac in cell lysate and cultured media of 7 osteosarcoma samples and 3 osteoblast samples.

and Neu5Ac, were significantly higher in the osteosarcoma than those in the osteoblast groups (Fig. 6, Table 3). There were no differences in both cell-associated and secreted KDN between those 2 groups. These results suggest that Neu5Gc and Neu5Ac increased in osteosarcoma and importantly only cell-associated forms were increased but not secreted forms. Thus increase in expression of cell-associated Neu5Gc, which humans are not able to synthesize, and Neu5Ac, a major Sia found in humans, might be involved in the pathology of osteosarcoma.

\section{Correlation of increased cell-associated Neu5Gc and Neu5Ac amounts with the transcriptional expression of osteogenic markers}

The significant increases of cell-associated Neu5Gc and Neu5Ac amounts in osteosarcoma compared to those in osteoblast cells might indicate their relation with the pathogenesis of osteosarcoma. Thus we investigated the correlation of the amounts of cell-associated Neu5Gc or Neu5Ac and levels of transcriptional expressions of the metastatic marker (MMP-9), the cartilage marker ( $\mathrm{Col} \mathrm{X}$ ), and the osteogenic markers (Col I, ON, BSP) in each osteosarcoma case. As shown in Fig. 7, the amount of cellassociated Neu5Gc seemed to be negatively corre- lated with the expression of the osteogenic markers, Col I, ON and BSP with $R^{2}$ values $0.414,0.720$ and 0.328 , respectively. Moreover, the amount of cell-associated Neu5Ac also showed a correlation with the expression of ON and BSP with $R^{2}$ values 0.487 and 0.545 , respectively. These results suggest that increased levels of cell-associated Neu5Gc and Neu5Ac might be involved with the pathology of osteosarcoma in terms of the reduction of the osteogenic ability as indicated by the negative correlation with those osteogenic markers. However, greater numbers of cases should be investigated to ensure this result.

\section{DISCUSSION}

Sia occupies the terminal position of the glycan chains of glycoconjugates, and plays an important role in various biological processes. It functions as a mediator for ligand-receptor and cell-cell interactions in fertilization, differentiation, immunological and neurological events ${ }^{1}$. In humans, the most abundant Sia forms are Neu5Ac and Neu5Gc. Owing to a human specific inactivating mutation in the CMP-Neu5Ac hydroxylase gene, humans are incapable of synthesizing Neu5 $\mathrm{Gc}^{3}$, and, therefore, in normal human tissues and fluids, Neu5Gc is usually detected in trace amounts which are thought to have 


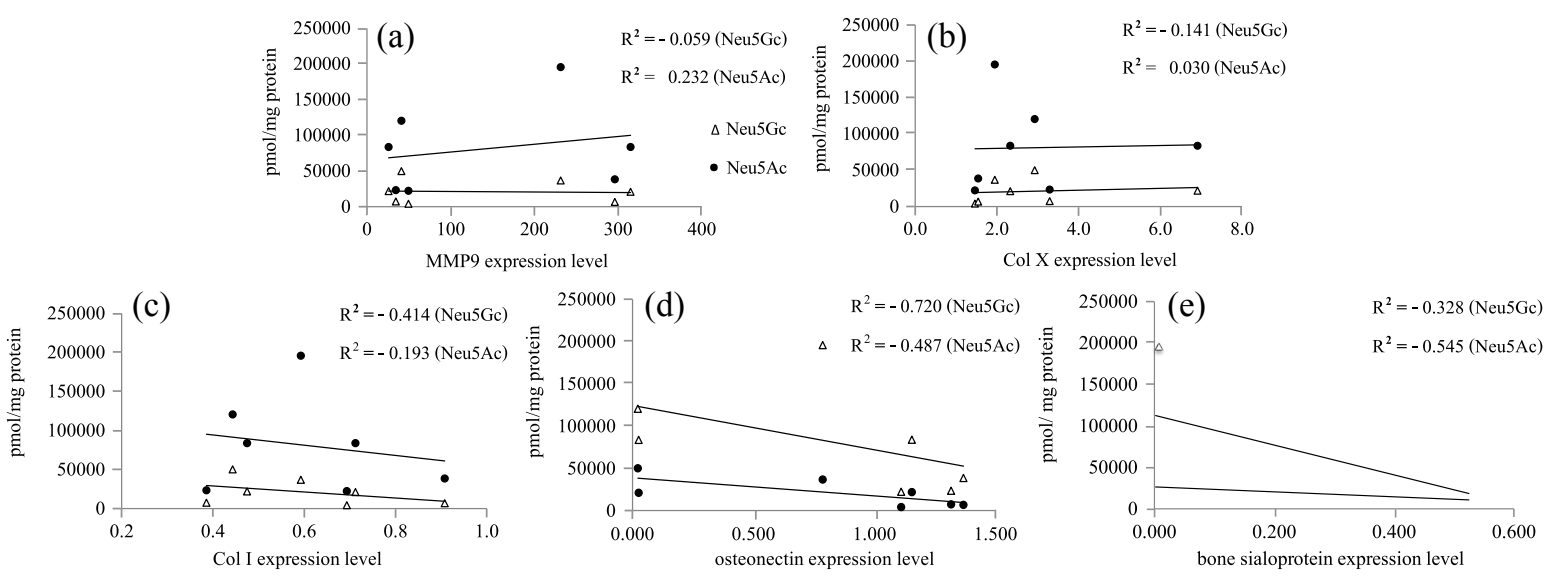

Fig. 7 Correlation of cell-associated Neu5Gc or Neu5Gc level and transcriptional expression levels of (a) MMP-9, (b) Col X, (c) Col I, (d) ON, and (e) BSP.

originated from exogenous sources ${ }^{4,5}$, and penetrates into the cell through a specific transporter, sialin.

Sia, the outermost residue of glycolipids and glycoproteins, has been found to alter its level and distribution in carcinogenetic processes with changes in cell membranes and organelles of the cancer cells. This alteration may result in deregulation of cell growth and proliferation. There is a correlation between high levels of Sia among different types of cancers ${ }^{26-30}$. Neu5Ac is present in the majority of Sia-containing tumours in humans, even though emerging data suggests that Neu5Gc is also correlated with certain human cancers $^{31,32}$. Neu5Gc, a nonhuman Sia, is abundantly present in malignant tissues ${ }^{33}$. The increase and metabolic incorporation of Neu5Gc are associated with inflammation within a mechanism of interaction with circulating anti-Neu5Gc antibodies ${ }^{34-37}$. Not only Neu5Ac and Neu5Gc increase in expression in malignant tissues, but KDN-containing $N$-glycan also accumulates in significant amounts in prostate cancer tissues ${ }^{9}$. Altogether the alteration of Sia expression and the distribution might be involved with the pathogenesis of cancer. Bull et al found that fluorinated Sia analogue (P-3Fax-Neu5Ac), which could block the synthesis of sialoglycans in murine melanoma cells, revealed clear consequences on cell adhesion, migration, and growth in vivo ${ }^{14}$.

Osteosarcoma is a rare malignant bone tumour most commonly occurring in children and young adults presenting with painful swelling. Although there have been significant advances in treatment over the past decades for osteosarcoma, nevertheless, it remains a poor-prognosis tumour ${ }^{38}$.
Treatment of pre-osteoblastic cells with $\alpha 2-3$ neuraminidase with the purpose of removal of $\alpha 2-$ 3 sialic acid from the cell surface resulted in the inhibition of osteogenesis ${ }^{39}$. This report showed a close relationship of Sia in regulating osteogenic differentiation. On the other hand, the level and distribution of Sia in two types of osteosarcoma cell lines has been reported. It was found that MG-63, a high metastatic cell line, produced up to 5 -fold more total Sias as compared with the Saos- 2 cells, a low metastatic cell line. The main cell-associated Sia found in MG-63 was Neu5Gc, whereas that from the secreted form was Neu5Ac. This indicated that the human osteosarcoma cells expressed both forms of Sia-containing glycoconjugates and the differences in the amounts of each of the two major Sia types and their distribution may be related to the morphology and/or metastatic of osteosarcoma ${ }^{15}$. Another studies of Sia level in the serum reported that the amount of Sia in the serum of osteosarcoma patients was higher than in the control group, but did not differ from those of other bone-forming groups ${ }^{16}$. However, the accumulated data on the level and distribution of Sia in osteosarcoma is still limited.

Here, we isolated osteosarcoma cells from 7 patients diagnosed with osteosarcoma and osteoblast cells from 3 patients diagnosed with other bone diseases. Isolated cells were investigated for their characteristics by the evaluation of the expression levels of osteogenic markers including Col I, ON, BSP, and the ability to synthesize the mineralized matrix by measurement of ALP activity and staining with Alizarin red S. Isolated osteosarcoma cells of all 7 patients showed low expression levels of 
osteogenic markers but the expression of cartilage marker, Col X, and the metastatic marker, MMP-9, were found to be highly expressed when compared to the levels of expression in isolated osteoblasts. Moreover, all cases of osteosarcoma showed low ability to produce mineralized matrix, while all cases of osteoblasts showed a high ability. This data indicates that the isolated cells were osteosarcoma and osteoblasts according to their diagnosis. Total Sia level in osteosarcoma was about twice that in the osteoblasts. The most abundant type was Neu5Ac in both cell-associated and secreted forms followed by Neu5Gc. This data shows different results when compared with the findings in the osteosarcoma cell line, MG-63, which was isolated from patients with different nationality with altered chromosomes ${ }^{15}$. Trace amounts of KDN was also detectable in both osteosarcomas and osteoblasts. Nevertheless, there were no differences in both cell-associated KDN and secreted KDN between the two groups. Interestingly, only cell-associated Neu5Ac and Neu5Gc, but not the secreted form, were found to have significantly increased in osteosarcoma compared to the levels in the osteoblast. This finding suggested that there might be an alteration of cell-associated Neu5Ac and Neu5Gc levels in osteosarcoma and this alteration might be related to its pathogenesis. However, more samples should be collected to confirm this result. Moreover, the correlation of increased Sia levels (cell-associated Neu5Ac and Neu5Gc) with the expression level of the osteogenic markers in each sample was also investigated. The increase of cell-associated Neu5Gc seemed to negatively correlate with the expression levels of the osteogenic markers, Col I, ON, and BSP, with $R^{2}$ values $0.414,0.720$, and 0.328 , respectively. The amount of cell-associated Neu5Ac also showed a negative correlation with the expression of $\mathrm{ON}$ and BSP, with $R^{2}$ values 0.487 and 0.545 , respectively. It was suggested that the increased Neu5Ac and Neu5Gc levels might be involved in osteosarcoma pathogenesis, with the reduction of osteogenic ability. However, the amounts of Neu5Ac and Neu5Gc did not correlate with the metastatic marker, MMP9. This data did not relate with the hypothesis that Sia might be related to metastatic ability of tumours due to its highly negative charge.

Since Sia was measured after hydrolysis with $0.1 \mathrm{~N} \mathrm{TFA}$, the free and glycoconjugated forms of Sia were not partly quantified. They should be quantified partly to identify the type of increased Sia in osteosarcoma cells. An increase of cell-associated Neu5Ac and Neu5Gc might be due to an alteration of the synthetic enzyme in the Sia synthetic pathway or an alteration of the Sia transporters for exogenous Sia, sialin. Thus the involvement of enzymes or transporters should be further identified as a potential target for the inhibition of osteosarcoma pathogenesis.

This study showed that the level and distribution of Sias osteosarcoma and osteoblast cells isolated from bone samples of patients with osteosarcoma and other bone diseases were different. Cell-associated Neu5Gc (nonhuman Sia) and Neu5Ac were found to be up-regulated in osteosarcomas when compared to those in the osteoblasts. Whereas there were no differences in cell-associated KDN levels, the levels of secreted Neu5Gc, Neu5Ac, and KDN between those two groups. These increases of the cell-associated Neu5Gc and Neu5Ac were negatively correlated with the expression of the osteogenic markers, but did not correlate with the metastatic marker, MMP-9. The increased cellassociated Neu5Gc and Neu5Ac might be involved with the osteogenic ability of osteosarcoma but not with the metastatic ability. However, more samples should be included and the pertinent mechanisms should be further investigated.

Acknowledgements: This study was supported by the Faculty of Medicine Research Fund, Chiang Mai University, Thailand (to TP) for all experimental costs. All experiments were performed in Thailand Excellence Centre for Tissue Engineering and Stem Cells, Faculty of Medicine, Chiang Mai University, Thailand. Sialic acid standards were kindly donated by Ken Kitajima, PhD., Nagoya University, Japan.

\section{REFERENCES}

1. Angata T, Varki A (2002) Chemical diversity in the sialic acids and related $\alpha$-keto acids: an evolutionary perspective. Chem Rev 102, 439-70.

2. Schauer R (2004) Sialic acids: fascinating sugars in higher animals and man. Zoology 107, 49-64.

3. Chou HH, Takematsu H, Diaz S, Iber J, Nickerson E, Wright KL, et al (1998) A mutation in human CMPsialic acid hydroxylase occurred after the Homo-Pan divergence. Proc Natl Acad Sci USA 95, 11751-6.

4. Bardor M, Nguyen DH, Diaz S, Varki A (2005) Mechanism of uptake and incorporation of the non-human sialic acid $N$-glycolylneuraminic acid into human cells. $J$ Biol Chem 280, 4228-37.

5. Tangvoranuntakul P, Gagneux P, Diaz S, Bardor M, Varki N, Varki A, et al (2003) Human uptake and incorporation of an immunogenic nonhuman dietary sialic acid. Proc Natl Acad Sci USA 100, 12045-50. 
6. Dao TL, Ip C, Patel J (1980) Serum sialyltransferase and 5 '-nucleotidase as reliable biomarkers in women with breast cancer. J Natl Canc Inst 65, 529-34.

7. Shamberger RJ (1984) Serum sialic acid in normals and in cancer patients. $J$ Clin Chem Clin Biochem 22, 647-51.

8. Yabu M, Korekane H, Takahashi H, Ohigashi H, Ishikawa O, Miyamoto Y (2013) Accumulation of free Neu5Ac-containing complex-type $N$-glycans in human pancreatic cancers. Glycoconj J 30, 247-56.

9. Yabu M, Korekane H, Hatano K, Kaneda Y, Nonomura N, Sato C, Kitajima K, Miyamoto Y (2013) Occurrence of free deaminoneuraminic acid (KDN)-containing complex-type $N$-glycans in human prostate cancers. Glycobiology 23, 634-42.

10. Levene PA, Landsteiner K (1927) On some new lipids. $J$ Biol Chem 75, 607-12.

11. Walz E (1927) Über das Vorkommen von Kerasin in der normalen Rindermilz. Hoppe-Seyler's Z Physiol Chem 166, 210-22.

12. Falconer RA, Errington RJ, Shnyder SD, Smith PJ, Patterson LH (2012) Polysialyltransferase: a new target in metastatic cancer. Curr Canc Drug Targets 12, 925-39.

13. Al-Saraireh YM, Sutherland M, Springett BR, Freiberger F, Ribeiro Morais G, Loadman PM, et al (2013) Pharmacological inhibition of polysialyltransferase ST8SiaII modulates tumour cell migration. PLOS ONE 8, e73366.

14. Bull C, Boltje TJ, Wassink M, de Graaf AM, van Delft FL, den Brok MH, et al (2013) Targeting aberrant sialylation in cancer cells using a fluorinated sialic acid analog impairs adhesion, migration, and in vivo tumor growth. Mol Canc Therapeut 12, 1935-46.

15. Tzanakakis GN, Nikitovic D, Katonis P, Kanakis I, Karamanos NK (2007) Expression and distribution of $N$-acetyl and $N$-glycolylneuraminic acids in secreted and cell-associated glycoconjugates by two human osteosarcoma cell lines. Biomed Chrom 21, 406-9.

16. Sandhu R, Lal H, Kundu ZS, Kharb S (2011) Serum fluoride and sialic acid levels in osteosarcoma. Biol Trace Elem Res 144, 1-5.

17. Martins AM, Pham QP, Malafaya PB, Sousa RA, Gomes ME, Raphael RM, et al (2009) The role of lipase and $\alpha$-amylase in the degradation of starch/poly( $\varepsilon$-caprolactone) fiber meshes and the osteogenic differentiation of cultured marrow stromal cells. Tissue Eng A 15, 295-305.

18. Gregory CA, Gunn WG, Peister A, Prockop DJ (2004) An Alizarin red-based assay of mineralization by adherent cells in culture: comparison with cetylpyridinium chloride extraction. Anal Biochem 329, 77-84.

19. Livak KJ, Schmittgen TD (2001) Analysis of relative gene expression data using real-time quantitative PCR and the $2^{-\Delta \Delta C_{\mathrm{T}}}$ method. Methods 25, 402-8.

20. Pautke C, Schieker M, Tischer T, Kolk A, Neth P,
Mutschler W, et al (2004) Characterization of osteosarcoma cell lines MG-63, Saos-2 and U-2 OS in comparison to human osteoblasts. Anticanc Res 24, 3743-8.

21. Yamakawa N, Sato C, Miyata S, Maehashi E, Toriyama M, Sato N, et al (2007) Development of sensitive chemical and immunochemical methods for detecting sulfated sialic acids and their application to glycoconjugates from sea urchin sperm and eggs. Biochimie 89, 1396-408.

22. Benayahu D, Shur I, Marom R, Meller I, Issakov J (2002) Cellular and molecular properties associated with osteosarcoma cells. $J$ Cell Biochem 84, 108-14.

23. Nelissen JM, Torensma R, Pluyter M, Adema GJ, Raymakers RA, van Kooyk Y, et al (2000) Molecular analysis of the hematopoiesis supporting osteoblastic cell line U2-OS. Exp Hematol 28, 422-32.

24. Birch MA, Skerry TM (1999) Differential regulation of syndecan expression by osteosarcoma cell lines in response to cytokines but not osteotropic hormones. Bone 24, 571-8.

25. Bilbe G, Roberts E, Birch M, Evans DB (1996) PCR phenotyping of cytokines, growth factors and their receptors and bone matrix proteins in human osteoblast-like cell lines. Bone 19, 437-45.

26. Makatsori E, Fermani K, Aletras A, Karamanos NK, Tsegenidis T (1998) Screening of $N$-acylneuraminic acids in serum and tissue specimens of mouse C57BI with Lewis' lung cancer by high-performance liquid chromatography. J Chrom B Biomed Sci Appl 712, 23-9.

27. Makatsori E, Aletras A, Karamanos NK, Tsegenidis T (1999) Analysis of $N$-acetyl and $N$-glycolylneuraminic acid in rat serum and tissues with Walker 256 carcinoma by high-performance liquid chromatography. Biomed Chrom 13, 57-60.

28. Sillanaukee P, Ponnio M, Jaaskelainen IP (1999) Occurrence of sialic acids in healthy humans and different disorders. Eur J Clin Investig 29, 413-25.

29. Narayanan S (1994) Sialic acid as a tumor marker. Ann Clin Lab Sci 24, 376-84.

30. Zhang Y, Zhang XY, Liu F, Qi HL, Chen HL (2002) The roles of terminal sugar residues of surface glycans in the metastatic potential of human hepatocarcinoma. $J$ Canc Res Clin Oncol 128, 617-20.

31. Kawai T, Kato A, Higashi H, Kato S, Naiki M (1991) Quantitative determination of $N$-glycolylneuraminic acid expression in human cancerous tissues and avian lymphoma cell lines as a tumor-associated sialic acid by gas chromatography-mass spectrometry. Canc Res 51, 1242-6.

32. Tzanakakis GN, Syrokou A, Kanakis I, Karamanos NK (2006) Determination and distribution of $\mathrm{N}$ acetyl- and $N$-glycolylneuraminic acids in culture media and cell-associated glycoconjugates from human malignant mesothelioma and adenocarcinoma cells. Biomed Chrom 20, 434-9. 
33. Varki A (2010) Colloquium paper: uniquely human evolution of sialic acid genetics and biology. Proc Natl Acad Sci USA 107, 8939-46.

34. Hedlund M, Padler-Karavani V, Varki NM, Varki A (2008) Evidence for a human-specific mechanism for diet and antibody-mediated inflammation in carcinoma progression. Proc Natl Acad Sci USA 105, 18936-41.

35. Padler-Karavani V, Hurtado-Ziola N, Pu M, Yu H, Huang S, Muthana S, et al (2011) Human xenoautoantibodies against a non-human sialic acid serve as novel serum biomarkers and immunotherapeutics in cancer. Canc Res 71, 3352-63.

36. Pearce OMT, Laubli H, Verhagen A, Secrest P, Zhang J, Varki NM, et al (2014) Inverse hormesis of cancer growth mediated by narrow ranges of tumor-directed antibodies. Proc Natl Acad Sci USA 111, 5998-6003.

37. Samraj AN, Läubli H, Varki N, Varki A (2014) Involvement of a non-human sialic acid in human cancer. Front Oncol 4, 1-13.

38. Meyers PA, Gorlick R (1997) Osteosarcoma. Pediatr Clin N Am 44, 973-89.

39. Xu L, Xu W, Xu G, Jiang Z, Zheng L, Zhou Y, et al (2013) Effects of cell surface $\alpha 2-3$ sialic acid on osteogenesis. Glycoconj J 30, 677-85. 